\title{
Properties of a planar waveguide with Eu(III)-SWCNTs
}

\author{
Fang Xie ${ }^{\mathrm{a}}$, Ke-Jun Feng ${ }^{\mathrm{b}}$, Gui-qiang Diaoc ${ }^{\mathrm{c}}$, Na Qiang ${ }^{\mathrm{d}}$ and Hao Liange,
}

School of Chemistry and Materials Engineering, Huizhou University, Guangdong 516007, China

axiefang4498@126.com, bhndx@hotmail.com, cdguiqiang@126.com, dqiangna93@163.com, elianghao4498@126.com

${ }^{*}$ Corresponding author

Keywords: Europium, SWCNTs, Luminescence, planar waveguide.

Abstract. A planar waveguide with Eu(III)-singlewalled carbon nanotubes (SWCNTs) was prepared by spinning coating. Its properties have been investigated by fluorescence spectrum, near-field scanning optical microscopy (NSOM) and m-line method. The film thickness is $1.825 \mu \mathrm{m}$ and refractive index is 1.593 . The result can provide some basic information for the design of new optoelectronics devices.

\section{Introduction}

Carbon nanotubes (CNTs) have attracted much attention because of their unique structures and exceptional electronic and mechanical properties[1]. Singlewalled carbon nanotubes (SWCNTs) have also been shown to absorb infrared light and may have applications in the optics areas [2].Europium complexs have been regarded as attractive for use as luminescent materials because of their high and sharply spiked fluorescence emission efficiency, long lifetime, good stability[3]. Thus, the hybrid materials combining the luminescent features of europium complexs and the unique properties of CNTs have attracted a significant attention recently[4,5] .

In this study, a planar waveguide with Eu(III)-SWCNTs was fabricated by spinning coating. According to fluorescence spectrum, near-field scanning optical microscopy (NSOM) and m-line method, the properties of Eu(III)-SWCNTs planar waveguide has been investigated.

\section{Experimental}

$\mathrm{Eu}(\mathrm{III})-\mathrm{SWCNTs}$ has been prepared according to the procedure reported before [5]. The structure of $\mathrm{Eu}(\mathrm{III})-\mathrm{SWCNT}$ is shown in Fig.1

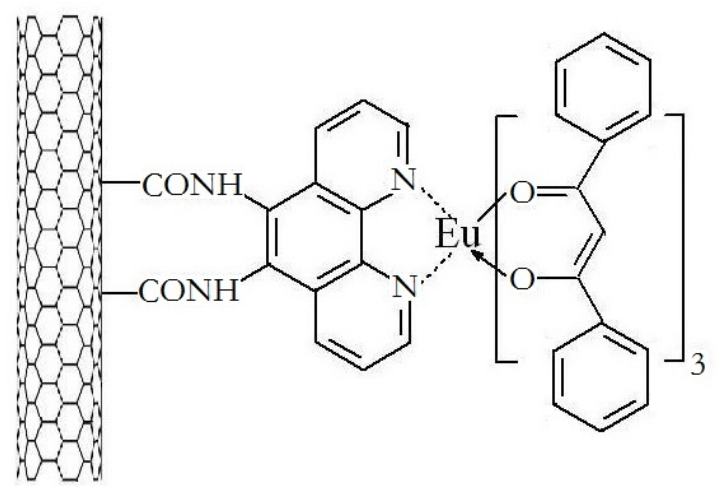

Fig. 1 Structure of Eu(III)-SWCNTs

0.1 mol of $4,4^{\prime}-$-Oxydianiline (ODA) is added into a solvent of $200 \mathrm{~g} \mathrm{~N}, \mathrm{~N}$-dimethylacetamide (DMAC) in a three-necked bottle at room temperature. After ODA dissolved completely, $0.1 \mathrm{~mol}$ of 4,4'-(hexafluoroisopropylidene)diphthalic anhydride(6FDA) is is added into the solution. After 6FDA dissolved completely, the solution is stirred for 12 hours at room temperature and the solution of polyamic acid is formed. To obtain the coating solution, Eu(III)-SWCNTs are added into the polyamic acid solution and dispersed by ultrasonic vibration for 30 minutes. Eu(III)-SWCNTs 
polyimide film was deposited on a substrate of fused silica $(3 \mathrm{~cm} \times 2 \mathrm{~cm})$ using spin coating. The refractive index of the fused silica at $650 \mathrm{~nm}$ is 1.459 . The sample was prepared at spin speed of 2000 revolution per minutes and dried in a vacuum oven for about 20 hours. After that, Eu(III)-SWCNTs planar waveguide was obtained.

The fluorescence emission spectrum of the planar waveguide was recorded on a Shimadzu RF-5301PC spectrofluorophotometer. A commercial near-field scanning optical microscopy (NSOM) from RHK Technology (USA) is used to detect the surface of the planar waveguide. The light from the $\mathrm{Ar}+$ ion laser $(457.9 \mathrm{~nm})$ is coupled into a very small aluminum-coated quartz single-mode glass fiber tip with a $50 \mathrm{~nm}$ aperture. The tip illustrates the sample and scans near the sample surface of interest. Typical pixel dwell time in our images was $10 \mathrm{~ms}$. Images were acquired and processed by using the software SPM32 5.06 version (RHK, USA). The refractive index $n_{1}$ and the thickness $h$ of the planar waveguide were obtained by the m-line method [7].

\section{Results and Discussion}

Fluorescence emission spectrum of Eu(III)-SWCNTs planar waveguide

The fluorescence emission spectra of Eu(III)-SWCNTs planar waveguide is shown in Fig.2.

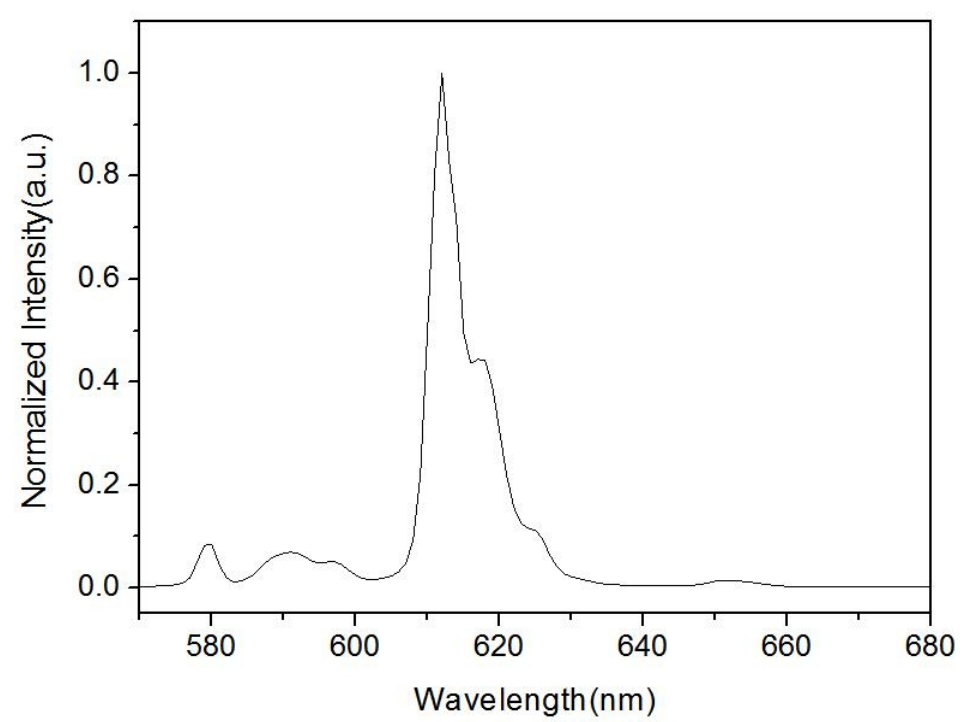

Fig.2 Fluorescence emission spectra of Eu(III)-SWCNTs planar waveguide

The emission spectrum was recorded form $570 \mathrm{~nm}$ to $680 \mathrm{~nm}$ under the excitation at $360 \mathrm{~nm}$. It can be found five emission peaks corresponding to $5 \mathrm{D} 0 \rightarrow 7 \mathrm{~F} 0,1,2,3,4$ can be clearly distinguished for Eu complex in solution. The emission spectrum is similar to those of other Eu complexes, which indicates that the energy levels of the central Eu ions are not affected obviousily by the SWCNTs.

\section{M-line measurement of Eu(III)-SWCNTs planar waveguide}

To investigate the basic parameters of Eu(III)-SWCNTs planar waveguide, its mode structure was measured by the m-line method. The incident polarized light is $650 \mathrm{~nm}$ wavelength from a diode laser. Fig. 3 is the experiment results of m-line method. 


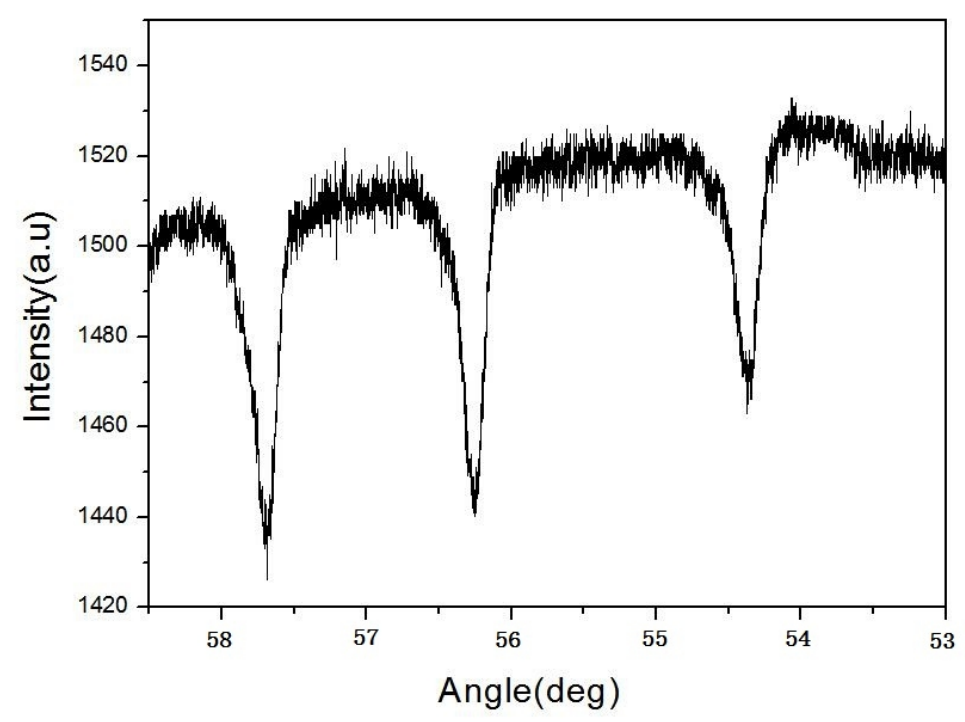

Fig.3 Experiment results of m-line method.

In Fig.3, there are four dropping peaks at $\theta_{1}(\mathrm{deg})=54.401, \theta_{2}(\mathrm{deg})=56.262$ and $\theta_{3}(\mathrm{deg})=57.692$, according to reference [8], the film thickness $h=1.825 \mu \mathrm{m}$ and refractive index $\mathrm{n}_{1}=1.593$ can be obtained. The results indicated that the optical signals can be transmitted in Eu(III)-SWCNTs planar waveguide.

Near-field scanning optical microscopy of Eu(III)-SWCNTs planar waveguide

Near-field scanning optical microscopy (NSOM) has the ability to achieve a high spatial resolution and measure the surface topography without any damage to the sample. The experimental setup is the same as reference [9]. Fig.4 is the near-field topography of Eu(III)-SWCNTs planar waveguide in an area of $10 \mu \mathrm{m} \times 10 \mu \mathrm{m}$.

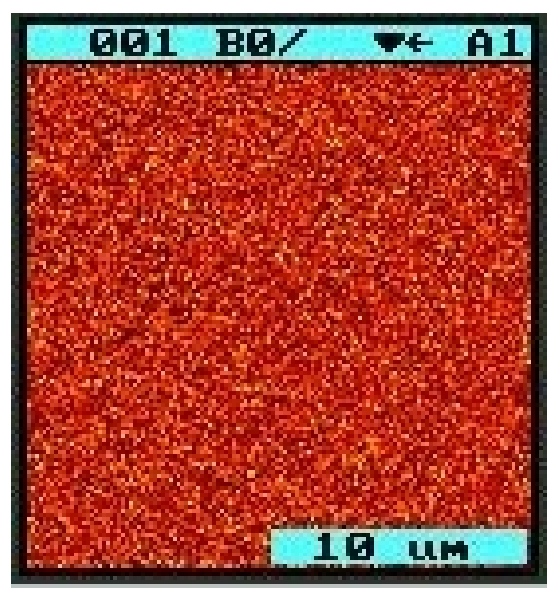

Fig.4 Near-field topography of Eu(III)-SWCNTs planar waveguide.

From Fig.4, it can be found that the film is homogenous under the resolution of 50nm and $\mathrm{Eu}(\mathrm{III})-\mathrm{SWCNTs}$ dispersed homogeneously in the polyimide matrix.

\section{Conclusions}

In conclusion, Eu(III)-SWCNTs planar waveguide was fabricated. The emission spectrum showed that the energy levels of the central Eu ions are not affected obviousily by the SWCNTs. The obtained film thickness of $\mathrm{Eu}(\mathrm{III})-\mathrm{SWCNTs}$ planar waveguide is $1.748 \mu \mathrm{m}$ and refractive index is 1.571 . 
Near-field scanning optical microscopy shows Eu(III)-SWCNTs dispersed homogeneously in the polyimide matrix.

\section{Acknowledgements}

This research was financially supported by the Natural Science Foundation of Guangdong Province, China (2014A030313642).

\section{References}

[1] J. Zhao, X.Chen and J.R.H.Xie: Analytica Chimica Acta Vol. 568 (2006) , p. 161

[2] J.Mohanraj and N.Armaroli: J. Phys. Chem. Lett. Vol. 4(2013) , p. 767

[3] G.E.Khalil, K.Lau, G.D.Phelan, B.Carlson, M.Gouterman, J.B.Callis and L.R.Dalton: Rev.Sci. Instrum. Vol. 75(2004), p. 192

[4] L.Maggini, J.A.Jensen, G.L.Re, J.M.Raquez,P.Dubois and D.Bonifazi: CARBON Vol.70 (2014), p. 22

[5] X.Xin, M.Pietraszkiewicz, O.Pietraszkiewicz, O.Chernyayeva, T.Kalwarczyk, E.Gorecka, D.Pociecha, H.Li and R.Hołyst: CARBON Vol. 50 (2012), p. 436

[7] H.Liang, B.Chen, J.Xu, W.Su, F.Guo, B.Guo, Q.Zhang, Z.Zheng and H.Ming:Materials Letters Vol. 59 (2005) , p. 4030

[8] R.Ulrich and R.Torge: J.Appl.Opt. Vol. 12 (1973), p. 2901

[9] H.Liang, F.Xie, X.Ren and Y.Chen: Adv. Mater. Res. Vol. 750-752(2013) , p. 836 